\title{
ALGEBRAIC PROPERTIES OF THE LEFSCHETZ ZETA FUNCTION, PERIODIC POINTS AND TOPOLOGICAL ENTROPY
}

Josefina Casasayas, Jaume llibre and Ana Nunes

\begin{abstract}
The Lefschetz zeta function associated to a continuous self-map $f$ of a compact manifold is a rational function $P / Q$. According to the parity of the degres of the polinomials $P$ and $Q$, we analize when the set of periodic points of $f$ is infinite and when the topological entropy is positive.
\end{abstract}

\section{Introduction}

In dynamical systems, it is often the case that algebraic information can be used to study qualitative and quantitative properties of the system. In this paper we study the dynamical consequences of simple algebraic properties of the Lefschetz zeta function $Z_{f}(t)$ associated to a continuous self-map $f: M \rightarrow M$ of a compact manifold $M$, which is always a rational function, i.e. $Z_{f}(t)=P(t) / Q(t)$ where $P(t)$ and $Q(t)$ are polynomials. We show that there is a relation between the parity of the degrees of $P(t)$ and $Q(t)$ and the finiteness of the set of periodic points of $f$ on one hand, and vanishing topological entropy on the other.

In Section 2 we shall review the definition and some basic propertics of the Lefschetz zeta function associated to a self-map and, in particular, give sufficient conditions for $P(t)$ and $Q(t)$ to have a finite factorization in cyclotomic polynomials. The statements and proofs of the results are given in Section 3.

The authors are deeply indebted to the late Professor Pere Menal who patiently taught us the algebra that we came upon trying to prove the results of this paper and the ones of [CLN]. 


\section{Lefschetz zeta function and cyclotomic polynomials}

Given a continuous self-map $f$ of a compact manifold $M$ of dimension $n$, its Lefschetz number is defined as

$$
L(f)=\sum_{k=0}^{n}(-1)^{k_{\mathrm{tr}}}\left(f_{* k}\right)
$$

where $f_{* k}: H_{k}(M ; \mathrm{Q}) \rightarrow H_{k}(M ; \mathrm{Q})$ is the endomorphism induced by $f$ on the $\mathrm{k}$-th rational homology group of $M$. The Lefschetz fixed point theorem says that if $L(f) \neq 0$ then $f$ has a fixed point. For the purpose of studying the whole set of periodic points of $f$, it is useful to consider the Lefschetz zeta function

$$
Z_{f}(t)=\exp \left(\sum_{m=1}^{\infty} \frac{L\left(f^{m}\right)}{m} t^{m}\right),
$$

which is a generating function for the Lefschetz numbers of all iterats of $f$ and can be computed from the homological endomorphisms $f_{* k}$ of $f$ as follows

$$
Z_{f}(t)=\prod_{k=0}^{n} \operatorname{det}\left(I_{j_{k}}-t f_{* k}\right)^{(-1)^{k+1}}
$$

where $j_{k}=\operatorname{dim}_{\mathrm{Q}} H_{k}(M ; \mathrm{Q})$, see [F1] for more details.

The following theorem is due to Fried (see Theorem 6 of [F2]).

Theorem 2.1. Let $M$ be a compact manifold and $f: M \rightarrow$ Int $(M)$ a $C^{1}$ map with finitely many periodic points. Then $Z_{f}(t)$ has a finite factorization in terms of the form $\left(1 \pm t^{T}\right)^{ \pm 1}$ with $r \in \mathrm{N}$.

As usual, we shall use the notation $c_{n}(t)$ for the $n$-th cyclotomic polynomial defined by

$$
c_{z_{z}}(t)=\frac{1-t^{n}}{\prod_{d \mid n, d<n} c_{d}(t)}
$$

for $n \in N \backslash\{1\}$ and $c_{1}(t)=1-t$.

Noticc that all the zeros of $c_{n}(t)$ are roots of unity.

A proof of the next proposition may be found in $[\mathrm{L}]$. 
Proposition 2.2. Let $\xi$ be a primitive $n$-th root of unity and $P(t)$ a polynomial with rational coefficients. If $P(\xi)=0$ then $c_{n}(t) \mid P(t)$.

Clearly, the degree $\varphi(n)$ of $c_{n}(t)$ verifies

$$
n=\sum_{d \mid n} \varphi(d)
$$

and so $\varphi(n)$ is the Euler function, which may be computed through

$$
\varphi(n)=\prod_{i=1}^{k} p_{i}^{\alpha_{i}-1}\left(p_{i}-1\right)
$$

where $n=p_{1}^{\alpha_{1}} \ldots p_{k}^{\alpha_{k}}$ is the prime decomposition of $n$. We remark that $\varphi(n)$ is even for $n>2$, and $\varphi(1)=\varphi(2)=1$.

Proposition 2.3. Let $M$ be a compact manifold and $f: M \rightarrow$ Int $(M)$ a $C^{1}$ map with finitely many periodic points. Then $Z_{f}(t)$ may be written in the form

$$
Z_{f}(t)=\frac{\prod_{i=1}^{R} c_{r_{2}}(t)}{\prod_{i=1}^{S} c_{s_{2}}(t)} .
$$

Proof: From Theorem 2.1, $Z_{j}(t)$ is a rational function $P(t) / Q(t)$ and all the roots of $P(t)$ and $Q(t)$ are roots of unity. Hence, by Proposition 2.2 , the result follows.

Now we shall show that the previous result holds for continuous surface maps with zero topological entropy. As usual we denote by $h(f)$ the topological entropy of a continuous map $f$ defined on a compact metric space.

Proposition 2.4. Let $M$ be a compact connected surface and $f$ : $M \rightarrow M$ a continuous map with zero topological entropy. Then $Z_{f}(t)$ may be written in the form

$$
Z_{f}(t)=\frac{\prod_{i=!}^{R} c_{r_{i}}(t)}{\prod_{i=1}^{S} c_{s_{i}}(t)} .
$$

Proof: If we prove that all the non-zero eigenvalues of $f_{* k}, k=0,1,2$; are roots of unity, then the result follows from (2.1) and Proposition 2.2.

Since $f_{* 0}$ is the identity, the unique eigenvalue of $f_{* 0}$ is 1 . Let us consider now $f_{* 2}$. If $M$ is non-orientable, then 0 is the only eigenvalue 
of $f_{* 2}$ because $H_{2}(M ; \mathrm{Q}) \approx 0$. If $M$ is orientable, $f_{* 2}(1)$ is the degree $D$ of $f$. From [MP] we know that if $|D|>1$ then $h(f) \geq \log |D|$. Hence, if $h(f)=0,|D| \leq 1$ and so the only possible eigenvalues for $f_{* 2}$ are $-1,0$ and 1 .

Finally, consider $f_{* 1}$. By Theorem 2 of $[\mathrm{M}]$, if $h(f)=0$ then all the eigenvalues $\lambda$ of $f_{* 1}$ satisfy $|\lambda| \leq 1$. We claim that every non-zero eigenvalue $\lambda$ of $f_{* 1}$ has modulus 1 . Let $\lambda_{1}, \ldots, \lambda_{k}$ be the non-zero eigenvalues of $f_{* 1}$. Then

$$
\operatorname{det}\left(I-t f_{* 1}\right)=\operatorname{det}\left(-t\left(f_{* 1}-t^{-1} I\right)\right)=(-1)^{k} \prod_{i=1}^{k}\left(\lambda_{i} t-1\right) .
$$

Moreover (2.2) must be a polynomial with integer coefficients, because $f_{* 1}$ is an integral matrix. Hence, in particular, $\prod_{i=1}^{k} \lambda_{i}$ must belong to Z. Therefore, $\prod_{i=1}^{k}\left|\lambda_{i}\right| \geq 1$, and the claim follows because $\left|\lambda_{i}\right| \leq 1$.

In short, (2.2) is a polynomial with integer coefficients, constant term \pm 1 and all its roots have modulus 1 . By a standard result in algebra (see Lemma 6.1 of [W]) the proposition follows.

\section{Main results}

Let $R(t)$ be a polynomial. We define $R^{*}(t)$ by

$$
R(t)=(1-t)^{\alpha}(1+t)^{\beta} R^{*}(t),
$$

where $\alpha$ and $\beta$ are non-negative integers such that $1-t$ and $1+t$ do not divide $R^{*}(t)$.

Theorem 3.1. Let $f: M \rightarrow$ Int $(M)$ be a $C^{1}$ map on the compact manifold $M$ and let $P(t) / Q(t)$ be its Lefschetz zeta function. If $P^{*}(t)$ or $Q^{*}(t)$ has odd degree, then $f$ has infinitely many periodic points.

Proof: Suppose that $f$ has finitely many periodic points. By Proposition 2.3, $P^{*}(t)$ and $Q^{*}(t)$ factorize into a product of cyclotomic polynomials $c_{m}(t)$ with $m>2$. So the degrees of $P^{*}(t)$ and $Q^{*}(t)$ are even, and the theorem follows.

Theorem 3.2. Let $f: M \rightarrow M$ be a continuous map on the compact connected surface $M$ and let $P(t) / Q(t)$ be its Lefschetz zeta function. If $P^{*}(t)$ or $Q^{*}(t)$ hos odd degree, then $f$ has positive topological entropy.

Proof: Suppose that $f$ has zero topological entropy. Then the theorem follows from Proposition 2.4 using the same arguments of the proof of Theorem 3.1. 
Theorem 3.3. Let $T^{n}$ be the n-dimensional torus and let $f: T^{n} \rightarrow$ $T^{n}$ be a continuous map with associated Lefschetz zeta function $P(t) /$ $Q(t)$. If $P^{*}(t)$ or $Q^{*}(t)$ has odd degree, then $f$ has infinitely many (least) periods.

Proof: Suppose that $f$ has finitely many periods. Then, by Theorem 4.1 and the proof of Corollary 4.2 of [ABLSS] it follows that all the non-zero eigenvalues of $f_{* 1}$ are roots of unity.

By Kunneth's formula the whole homology vector space of $T^{n}$ is given as a tensor product of $H_{*}\left(S^{1} ; \mathrm{Q}\right)$ and so one obtains all the eigenvalues of $f_{* k}$ as a product of the eigenvalues of $f_{* 1}$. Since all the roots of $P(t)$ and $Q(t)$ are roots of unity, by Proposition 2.2, $P(t)$ and $Q(t)$ have a finite factorization in cyclotomic polynomials. Therefore, the result follows as in the proof of Theorem 3.1.

Theorem 3.4. Let $f: M \rightarrow M$ be a continuous map on a compact connected manifold of dimension $n$. Suppose that for $k=2, \ldots, n$ all the eigenvalues of $f_{* k}$ can be obtained as products of the eigenvalues of $f_{* 1}$. Let $P(t) / Q(t)$ be the Lefschetz zeta function of $f$. If $P^{*}(t)$ or $Q^{*}(t)$ has odd degree, then $f$ has positive topological entropy.

Proof: Suppose that $h(f)=0$. By the same argumcnt of the proof of Proposition 2.4 we have that all the non-zero eigenvalues of $f_{* 0}$ and $f_{* 1}$ are roots of unity. So, by hypothesis, $P(t)$ and $Q(t)$ have a finite factorization in cyclotomic polynomials. Hence, the result follows as in the proof of Theorem 3.1 .

Wc remark that the hypothesis that for $k=2, \ldots, n$ all the eigenvalues of $f_{* k}$ can be obtained as products of the eigenvalues of $f_{* 1}$ holds for continuous self-maps of $T^{n}$, and also for continuous maps of many Eilenberg-Mac Lane spaces.

\section{References}

[ABLSS] Ll. Alsedà, S. Baldwin, J. Llibre, R. Swanson And W. SZLENK, Minimal sets of periods for torus maps via Nielsen numbers, Preprint of the Centre de Recerca Matemàtica (1991).

[CLN] J. Casasayas, J. Limbre and A. Nunes, Periodic Orbits for Transversal Maps, Preprint of the Centre de Recerca Matemàtica (1991).

[F1] J. M. FRANKs, "Homology and Dynamical Systems," CBMS Regional Conf. Series 49, Amer. Math. Soc., 1982. 
[F2] D. FRIED, "Periodic points and twisted coefficients," in Lecture Notes in Maths. 1007, Springer Verlag, 1983, pp. $175 \cdots 179$.

[L] S. LANG, "Algebra," Addison Wesley, 1971.

[M] A. MANNING, "Topological entropy and the first homology group," Lecture Notes in Math. 468, Springer Verlag, 1975, pp. 185-199.

[MP] M. Misiurewicz AND F. PRZYTYCKI, Topological Entropy and degree of smooth mappings, Bulletin de l'Académie Polonaise des Sciences, Série des Sciences Math., Astr. et Phys. XXV (1977), $573-578$.

[W] L. C. Washington, "Introduction to Cyclotomic Fields," Springer Verlag, 1982.

Josefina Casasayas:

Departament de Matemàtica Aplicada

i Anàlisi

Universitat de Barcelona

Gran Via 585

08071 Barcelona

SPAIN
Jaume Llibre:

Departament de Matemàtiques

Universitat Autònoma de Barcelona

08193 Bellaterra (Barcelona)

SPAIN

Ana Nunes:

Departamento de Física

Universidade de Lisboa

Campo Grande, Ed C1, Piso 4

1700 Lisboa.

PORTUGAL

Rebut el 17 de Gener de 1992 